\title{
ULAM'S STABILITIES OF FRACTIONAL INTEGRO-DIFFERENTIAL EQUATIONS
}

\author{
Maher Nazmi Qarawani \\ Department of Mathematics \\ Al-Quds Open University \\ Salfit, West-Bank, PALESTINE
}

Abstract: In this paper we establish Hyers-Ulam and Hyers-Ulam-Rassias stability for fractional integrodifferential equations

$$
x^{(\alpha)}(t)=g(t, x(t))+\int_{t_{0}}^{t} G(t, s, x(s)) d s, \quad \alpha \in \mathbb{R}, 0<\alpha \leq 1,
$$

with the initial condition $x^{(\alpha-1)}\left(t_{0}\right)=x_{0}$.

AMS Subject Classification: 26A32, 34K20, 37C75.

Key Words: Hyers-Ulam-Rassias Stability, Fractional Equations, Integro-Differential Equations.

\section{Introduction}

The study of stability problems for various functional equations originated from a famous talk given by Ulam In 1940 (see [1]). In the talk, Ulam discussed a problem concerning the stability of homomorphisms. A significant breakthrough came in 1941, when Hyers [2] gave a partial solution to Ulam's problem.. In 1978, Rassias [3] provided a remarkable generalization of the Ulam-Hyers stability of mappings by considering variables. During the last two

$\begin{array}{lr}\text { Received: } & \text { September 8, } 2016 \\ \text { Revised: } & \text { December 13, } 2016 \\ \text { Published: } & \text { March 28, } 2017\end{array}$

(C) 2017 Academic Publications, Ltd. url: www.acadpubl.eu 
decades very important contirbutions to the stability problems of functional equations were given by many mathematicians (see [4-11]). A generalization of Ulam's problem was recently proposed by replacing functional equations with differential equations: The differential equation $F\left(t, y(t), y^{\prime}(t), \ldots, y^{(n)}(t)\right)=0$ has the Hyers-Ulam stability if for given $\varepsilon>0$ and a function $y$ such that

$$
\left|F\left(t, y(t), y^{\prime}(t), \ldots, y^{(n)}(t)\right)\right| \leq \varepsilon
$$

there exists a solution $y_{0}$ of the differential equation such that

$$
\left|y(t)-y_{0}(t)\right| \leq K(\varepsilon)
$$

and $\lim _{\varepsilon \rightarrow 0} K(\varepsilon)=0$.

Obloza seems to be the first author who has investigated the Hyers-Ulam stability of linear differential equations (see $[12,13]$ ). Thereafter, Alsina and Ger published their paper [14], which handles the Hyers-Ulam stability of the linear differential equation $y^{\prime}(t)=y(t)$ : If a differentiable function $\mathrm{y}(\mathrm{t})$ is a solution of the inequality $\left|y^{\prime}(t)-y(t)\right| \leq \varepsilon$ for any $t \in(a, \infty)$, then there exists a constant $c$ such that $\left|y(t)-c e^{t}\right| \leq 3 \varepsilon$ for all $t \in(a, \infty)$.Recently, the Hyers-Ulam stability problems of linear differential equations of first order and second order with constant coefficients were studied by using the method of integral factors (see $[15,16]$ ). The results given in [17-19] have been generalized by Popa and Rus $[20,21]$ for the linear differential equations of nth order with constant coefficients. For more details on Hyers-Ulam stability and the generalized Hyers-Ulam stability, we refer the reader to the papers [22-28].

Fractional calculus is a generalization of ordinary differentiation and integration to arbitrary order (non-integer). In recent years, fractional differential equations arise naturally in various fields such as rheology, fractals, chaotic dynamics, modeling and control theory, signal processing, bioengineering and biomedical applications, etc; Fractional derivatives provide an excellent instrument for the description of memory and hereditary properties of various materials and processes,[29-30]. Some researchers have used the fixed point approach to investigate the Hyers-Ulam stability for fractional differential equations [e.g. 31,33]. The objective of this article is to investigate the Hyers-Ulam-Rassias Stability for the stability and Hyers-Ulam Criteria for fractional integrodifferential equations

$$
x^{(\alpha)}(t)=g(t, x(t))+\int_{t_{0}}^{t} G(t, s, x(s)) d s, \quad \alpha \in \mathbb{R}, 0<\alpha \leq 1
$$


with the initial condition

$$
x^{(\alpha-1)}\left(t_{0}\right)=x_{0} .
$$

where $\mathbb{R}$ denotes the set of real numbers, $J=\left[t_{0}, t+a\right], a>0, g \in C[J \times \mathbb{R}, \mathbb{R}], G \in$ $C[J \times J \times \mathbb{R}, \mathbb{R}]$, and $x_{0}$ is a real constant.

\section{Preliminaries}

In this section, we give some basic definitions and Lemmas which we used to prove the main results.

Definition 1. Let $\mathrm{f}$ be a function which is defined on $[a, b]$. For $\alpha>0$, define

$$
I_{a}^{{ }^{b} \alpha} g(t)=\frac{1}{\Gamma(\alpha)} \int_{a}^{b}(b-s)^{\alpha-1} g(s) d s
$$

provided that this integral exists, where $\Gamma$ is the Gamma function.

Definition 2. Assume that for continuously differentiable functions $g$ : $J \rightarrow \mathbb{R}, G: J \times J \rightarrow \mathbb{R}$ and satisfying fractional differential inequality

$$
\left|x^{(\alpha)}(t)-g(t, x(t))-\int_{t_{0}}^{t} G(t, s, x(s)) d s\right| \leq \varepsilon,
$$

for all $t \in J$ and for each $\varepsilon>0$, where $x^{(\alpha)}(t)$ denotes the fractional drivative of order $\alpha$.there exists a solution $y_{0}: J \rightarrow Y$

of the fractional initial value problem (1.1)and (1.2) such that $\left|x(t)-x_{0}(t)\right| \leq$ $K \varepsilon$, for all $t \in J$. Then we say that the above fractional initial value problem (1.1)and (1.2) has the Hyers-Ulam stability.

Definition 3. We say that equation (1.1) with initial condition (1.2) has the Hyers-Ulam-Rassias stability with respect to $\varphi$ if there exists a positive constant $K>0$ with the following property:

For each $x(t)$ satisfying

$$
\left|x^{(\alpha)}(t)-g(t, x(t))-\int_{t_{0}}^{t} G(t, s, x(s)) d s\right| \leq \varphi(t)
$$

then there exists some solution $x_{0}(t)$ of the equation (1.1) with (1.2) such that $\left|x(t)-x_{0}(t)\right| \leq K \varphi(t)$. 
Lemma 1. [34] The IVP (1.1) and (1.2) is equivalent to the nonlinear integral equation

$$
\begin{aligned}
x(t)= & \frac{x_{0}}{\Gamma(\alpha)}\left(t-t_{0}\right)^{\alpha-1}+\frac{1}{\Gamma(\alpha)} \int_{t_{0}}^{t}(t-s)^{\alpha-1} g(s, x(s)) d s \\
& +\frac{1}{\Gamma(\alpha)} \int_{t_{0}}^{t}(t-s)^{\alpha-1} \int_{s}^{t} G(\tau, s, x(s)) d \tau d s,
\end{aligned}
$$

where $0<t_{0} \leq t \leq t_{0}+a$. In otherwords, every solution of the integral (2.3) is also a solution of the origina IIVP (1.1) and (1.2), and viceversa.

Lemma 2. (Gronwall's lemma). Let $\mathrm{u}(\mathrm{t})$ and $\mathrm{v}(\mathrm{t})$ be nonnegative continuous functions on some interval $0<t_{0} \leq t \leq t_{0}+a$. Also, let the function $f(t)$ be positive, continuous, and monotonically nondecreasing on $\left[t_{0}, t_{0}+a\right]$ and satisfy the inequality

$$
u(t) \leq f(t)+\int_{t_{0}}^{t} u(s) v(s) d s
$$

then, there holds the inequality

$$
u(t) \leq f(t) \exp \left(\int_{t_{0}}^{t} u(s) d s, \text { for } t_{0} \leq t \leq t_{0}+a\right.
$$

Proof. For the proof of Lemma 1.2, see [35].

\section{Main Results On Hyers-Ulam and Hyers-Ulam-Rassias Stability}

In this section, we will prove our main results, and establish the HU stability of solution of (1.1) satisfying (1.2).

Theorem 4. let the function $g$ satisfy the inequality

$$
|g(t, x(t))-g(t, y(t))| \leq \beta(t)|x-y|,
$$


and let $G$ satisfy the inequality

$\left|\int_{s}^{t}[G(\tau, s, x(s))-G(\tau, s, y(s))] d \tau\right| \leq \gamma(t)|x-y|, \quad s \in\left[t_{0}, t\right]$,

where $\beta(t)$ and $\gamma(t)$ are continuous and nonnegative functions such that

$$
\sup \int_{t_{0}}^{t}(t-s)^{\alpha-1}[(\beta(s)+\gamma(s)] d s<\infty
$$

Then the problem (1.1),(1.2) is stable in the sense of Hyers and Ulam.

Proof. Let $x(t) \in C[J, \mathbb{R}]$ be a solution of inequation (2.2) i.e.

$$
\left|x^{(\alpha)}(t)-g(t, x(t))-\int_{t_{0}}^{t} G(t, s, x(s)) d s\right| \leq \varepsilon,
$$

Applying the integral operator (2.1) to the inequality (3.9) we obtain

$$
\begin{aligned}
& \mid x(t)-\frac{x_{0}}{\Gamma(\alpha)}\left(t-t_{0}\right)^{\alpha-1}-\frac{1}{\Gamma(\alpha)} \int_{t_{0}}^{t}(t-s)^{\alpha-1} g(s, x(s)) d s \\
& -\frac{1}{\Gamma(\alpha)} \int_{t_{0}}^{t}(t-s)^{\alpha-1} \int_{s}^{t} G(\tau, s, x(s)) d \tau d s \mid \leq \frac{\varepsilon}{\Gamma(\alpha)}\left(t-t_{0}\right)^{\alpha-1}
\end{aligned}
$$

On can easily show that $z(t) \in C[J, \mathbb{R}]$ defined by

$$
\begin{aligned}
z(t)= & \frac{x_{0}}{\Gamma(\alpha)}\left(t-t_{0}\right)^{\alpha-1}+\frac{1}{\Gamma(\alpha)} \int_{t_{0}}^{t}(t-s)^{\alpha-1} g(s, z(s)) d s \\
& +\frac{1}{\Gamma(\alpha)} \int_{t_{0}}^{t}(t-s)^{\alpha-1} \int_{s}^{t} G(\tau, s, z(s)) d \tau d s
\end{aligned}
$$

satisfies the IVP (1.1),(1.2). 
Now we estimate the difference

$$
\begin{aligned}
|x(t)-z(t)| \leq \mid & x(t)-\frac{x_{0}}{\Gamma(\alpha)}\left(t-t_{0}\right)^{\alpha-1}-\frac{1}{\Gamma(\alpha)} \int_{t_{0}}^{t}(t-s)^{\alpha-1} g(s, x(s)) d s \\
& -\frac{1}{\Gamma(\alpha)} \int_{t_{0}}^{t}(t-s)^{\alpha-1} \int_{s}^{t} G(\tau, s, x(s)) d \tau d s \mid \\
& +\mid z(t)-\frac{x_{0}}{\Gamma(\alpha)}\left(t-t_{0}\right)^{\alpha-1}-\frac{1}{\Gamma(\alpha)} \int_{t_{0}}^{t}(t-s)^{\alpha-1} g(s, x(s)) d s \\
& -\frac{1}{\Gamma(\alpha)} \int_{t_{0}}^{t}(t-s)^{\alpha-1} \int_{s}^{t} G(\tau, s, x(s)) d \tau d s \mid
\end{aligned}
$$

Or equivalently,

$$
\begin{gathered}
|x(t)-z(t)| \leq \frac{\varepsilon}{\Gamma(\alpha)}\left(t-t_{0}\right)^{\alpha-1}+\frac{1}{\Gamma(\alpha)} \int_{t_{0}}^{t}(t-s)^{\alpha-1} \mid g(s, x(s))-g(s, z(s)) d s \\
\quad+\frac{1}{\Gamma(\alpha)} \int_{t_{0}}^{t}(t-s)^{\alpha-1} \int_{s}^{t}|G(\tau, s, x(s))-G(\tau, s, z(s))| d \tau d s \\
\left.\left.\leq \frac{\varepsilon}{\Gamma(\alpha)}\left(t-t_{0}\right)^{\alpha-1}+\frac{1}{\Gamma(\alpha)} \int_{t_{0}}^{t}(t-s)^{\alpha-1} \mid x(s)\right)-z(s)\right) \mid[\beta(t)+\gamma(t)] d s
\end{gathered}
$$

By Gronwell's lemma, we obtain

$$
|x(t)-z(t)| \leq \frac{\varepsilon}{\Gamma(\alpha)}\left(t-t_{0}\right)^{\alpha-1} \exp \left(\frac{1}{\Gamma(\alpha)} \int_{t_{0}}^{t}(t-s)^{\alpha-1}[\beta(t)+\gamma(t)] d s\right)
$$

In view of (3.3) there exists a positive constant $K$ such that

$$
|x(t)-z(t)| \leq K \varepsilon
$$

which completes the proof. 
Now we will prove the Hyers-Ulam-Rassias stability (HUR) of problem (1.1), (1.2).

Theorem 5. Let the function $g$ satisfy the inequality

$$
|g(t, x(t))-g(t, y(t))| \leq \beta(t)|x-y|,
$$

and let $G$ satisfy the inequality

$\left|\int_{s}^{t}[G(\tau, s, x(s))-G(\tau, s, y(s))] d \tau\right| \leq \gamma(t)|x-y|, \quad s \in\left[t_{0}, t\right]$,

where $\gamma(t)$ and $\beta(t)$ are continuous and nonnegative functions such that

$$
\sup \int_{t_{0}}^{t}(t-s)^{\alpha-1}[(\beta(s)+\gamma(s)] d s<\infty
$$

If $\varphi(t):[0, \infty) \rightarrow(0, \infty)$ is a continuous function such that

$$
\int_{t_{0}}^{t}(t-s)^{\alpha-1} \varphi(s) d s \leq C \varphi(t)
$$

then the problem $(1.1),(1.2)$ is stable in the sense of HUR.

Proof. Let $x(t) \in C[J, \mathbb{R}]$ be a solution of inequation (2.2) i.e.

$$
\left|x^{(\alpha)}(t)-g(t, x(t))-\int_{t_{0}}^{t} G(t, s, x(s)) d s\right| \leq \varphi(t)
$$

Applying the integral operator (2.1) to the inequality (3.9) we obtain

$$
\begin{gathered}
\mid x(t)-\frac{x_{0}}{\Gamma(\alpha)}\left(t-t_{0}\right)^{\alpha-1}-\frac{1}{\Gamma(\alpha)} \int_{t_{0}}^{t}(t-s)^{\alpha-1} g(s, x(s)) d s \\
-\frac{1}{\Gamma(\alpha)} \int_{t_{0}}^{t}(t-s)^{\alpha-1} \int_{s}^{t} G(\tau, s, x(s)) d \tau d s \mid \leq \frac{1}{\Gamma(\alpha)} \int_{t_{0}}^{t}(t-s)^{\alpha-1} \varphi(s) d s
\end{gathered}
$$


On can easily show that $z(t) \in C[J, \mathbb{R}]$ defined by

$$
\begin{aligned}
z(t)= & \frac{x_{0}}{\Gamma(\alpha)}\left(t-t_{0}\right)^{\alpha-1}+\frac{1}{\Gamma(\alpha)} \int_{t_{0}}^{t}(t-s)^{\alpha-1} g(s, z(s)) d s \\
& +\frac{1}{\Gamma(\alpha)} \int_{t_{0}}^{t}(t-s)^{\alpha-1} \int_{s}^{t} G(\tau, s, z(s)) d \tau d s
\end{aligned}
$$

satisfies the IVP (1.1),(1.2).

Now we estimate the difference

$$
\begin{aligned}
|x(t)-z(t)| \leq \mid & x(t)-\frac{x_{0}}{\Gamma(\alpha)}\left(t-t_{0}\right)^{\alpha-1}-\frac{1}{\Gamma(\alpha)} \int_{t_{0}}^{t}(t-s)^{\alpha-1} g(s, x(s)) d s \\
& -\frac{1}{\Gamma(\alpha)} \int_{t_{0}}^{t}(t-s)^{\alpha-1} \int_{s}^{t} G(\tau, s, x(s)) d \tau d s \mid \\
& +\mid z(t)-\frac{x_{0}}{\Gamma(\alpha)}\left(t-t_{0}\right)^{\alpha-1}-\frac{1}{\Gamma(\alpha)} \int_{t_{0}}^{t}(t-s)^{\alpha-1} g(s, x(s)) d s \\
& -\frac{1}{\Gamma(\alpha)} \int_{t_{0}}^{t}(t-s)^{\alpha-1} \int_{s}^{t} G(\tau, s, x(s)) d \tau d s \mid
\end{aligned}
$$

Using the conditions (3.5), (3.6) and (3.8) we infer that

$$
\begin{aligned}
|x(t)-z(t)| \leq & \frac{1}{\Gamma(\alpha)} \int_{t_{0}}^{t}(t-s)^{\alpha-1} \varphi(s) d s+\frac{1}{\Gamma(\alpha)} \int_{t_{0}}^{t}(t-s)^{\alpha-1} \mid g(s, x(s))-g(s, z(s)) d s \\
& +\frac{1}{\Gamma(\alpha)} \int_{t_{0}}^{t}(t-s)^{\alpha-1} \int_{s}^{t}|G(\tau, s, x(s))-G(\tau, s, z(s))| d \tau d s \\
\leq & \left.\left.\frac{C \varphi(t)}{\Gamma(\alpha)}+\frac{1}{\Gamma(\alpha)} \int_{t_{0}}^{t}(t-s)^{\alpha-1} \mid x(s)\right)-z(s)\right) \mid[\beta(t)+\gamma(t)] d s
\end{aligned}
$$


By Gronwell's lemma, we obtain

$$
|x(t)-z(t)| \leq \frac{C \varphi(t)}{\Gamma(\alpha)}\left(t-t_{0}\right)^{\alpha-1} \exp \left(\frac{1}{\Gamma(\alpha)} \int_{t_{0}}^{t}(t-s)^{\alpha-1}[\beta(t)+\gamma(t)] d s\right)
$$

According to (3.7) there exists a positive constant $K$ such that

$$
|x(t)-z(t)| \leq K \varepsilon
$$

which completes the proof.

In the following theorem we establish the Hyers-Ulam-Rassias stability for (1.1),

(1.2) in the interval $0 \leq t_{0} \leq t \leq \infty$.

Theorem 6. Let the function $g$ satisfy the inequality

$$
\mid g((t, x(t))-g((t, y(t))|\leq \beta(t)| x-y \mid,
$$

and let $G$ satisfy the inequality

$$
\left|\int_{s}^{t}[G(\tau, s, x(s))-G(\tau, s, y(s))] d \tau\right| \leq \gamma(t)|x-y|, \quad t_{0} \leq s \leq t \leq \infty
$$

where $\beta(t)$ and $\gamma(t)$ are continuous and nonnegative functions such that

$$
\sup _{t_{0} \leq t \leq \infty} \int_{t_{0}}^{t}(t-s)^{\alpha-1}[(\beta(s)+\gamma(s)] d s<\infty
$$

If $\varphi(t):[0, \infty) \rightarrow(0, \infty)$ is a continuous function such that

$$
\int_{t_{0}}^{\infty}(t-s)^{\alpha-1} \varphi(s) d s \leq C \varphi(t)
$$

then the problem (1.1),(1.2) is stable in the sense of HUR as $t \rightarrow \infty$.

Proof. Applying the same approach used in the Theorem 2, we can get the proof of theorem . 


\section{Conclusion}

In this work, the problem of the Hyers-Ulam and Hyers-Ulam-Rassias Stability of solution of Ulam's Stabilities of Fractional Integro-Differential Equations has been investigated and solved using the direct method.

\section{References}

[1] Ulam S. Problems in Modern Mathematics, John Wiley \& Sons (1964) New York, USA, Science edition.

[2] Hyers D. On the stability of the linear functional equation, Proceedings of the National Academy of Sciences of the United States of America, 27 (1941) 222-224.

[3] Rassias T On the stability of the linear mapping in Banach spaces, Proceedings of the American Mathematical Society, 72, 2 (1978) 297-300.

[4] Miura T., Takahasi S., Choda H., On the Hyers-Ulam stability of real continuous function valued differentiable map, Tokyo Journal of Mathematics 24, (2001) 467-476.

[5] Jung S, On the Hyers-Ulam-Rassias stability of approximately additive mappings, Journal of Mathematics Analysis and Application 204 (1996) 221-226.

[6] Park C., On the stability of the linear mapping in Banach modules, Journal of Mathematics Analysis and Application 275 (2002) 711-720.

[7] Gavruta P., A generalization of the Hyers-Ulam-Rassias stability of approximately additive mappings, Journal of Mathematical Analysis and Applications, 184, 3 (1994) 431436.

[8] Jun K., Lee Y., A generalization of the Hyers-Ulam-Rassias stability of the Pexiderized quadratic equations, Journal of Mathematical Analysis and Applications 297, 1 (2004) $70-86$.

[9] Jung S., Hyers-Ulam-Rassias Stability of Functional Equations in Mathematical Analysis, Hadronic Press, Palm Harbor, Fla, USA, (2001)

[10] Park C., Homomorphisms between Poisson JC*-algebras, Bulletin of the Brazilian Mathematical Society, 36, 1 (2005) 79-97.

[11] Park C., Cho Y. and Han M., Functional inequalities associated with Jordan-von Neumann type additive functional equations, Journal of Inequalities and Applications, Article ID 41820 (2007)13 p.

[12] Obloza M., Hyers stability of the linear differential equation, Rocznik Nauk.-Dydakt. Prace Mat., 13 (1993) 259-270.

[13] Obloza M, Connections between Hyers and Lyapunov stability of the ordinary differential equations, Rocznik Nauk.-Dydakt. Prace Mat., 14 (1997) 141-146.

[14] Alsina C., Ger R., On some inequalities and stability results related to the exponential function, Journal of Inequalities and Application 2 (1998) 373-380.

[15] Wang G., Zhou M. and Sun L, Hyers-Ulam stability of linear differential equations of first order, Applied Mathematics Letters 21 (2008) 1024-1028. 
[16] Li Y. and Shen Y, Hyers-Ulam Stability of Nonhomogeneous Linear Differential Equations of Second Order, International Journal of Mathematics and Mathematical Sciences, Article ID 576852, (2009) 7 .

[17] Jung S., Hyers-Ulam stability of a system of first order linear differential equations with constant coefficients, Journal of Mathematical Analysis and Applications 320, 2 (2006) 59-561.

[18] Rus I., Remarks on Ulam stability of the operatorial equations, Fixed Point Theory 10, 2 (2009) 305-320.

[19] Rus I, Ulam stability of ordinary differential equations, Studia Universitatis Babes-Bolyai: Mathematica, 5 (2009) 125-133.

[20] Popa D. and Rus I. On the Hyers-Ulam stability of the linear differential equation. Journal of Mathematical Analysis and Applications, 381, 2 (2011) 530-537.

[21] Popa D. and Rus I. Hyers-Ulam stability of the linear differential operator with nonconstant coefficients. Applied Mathematics and Computation 219, 4 (2012) 1562-1568.

[22] Tkahasi E., Miura T., and Miyajima S. On the Hyers-Ulam stability of the Banach spacevalued differential equation, Bulletin of the Korean Mathematical Society 39, 2 (2002) 309-315.

[23] Jung S., Hyers-Ulam stability of linear differential equations of first order, Journal of Mathematics Analysis and Application, 311, 1 (2005) 139-146.

[24] Miura T., Miyajima S., Takahasi S., A characterization of Hyers-Ulam stability of first order linear differential operators. Journal of Mathematics Analysis and Application 286 (2003) 136-146.

[25] Li Y. Hyers-Ulam Stability of Linear Differential Equations, Thai Journal of Mathematics 8, 2 (2010) 215-219.

[26] Gavruta P., Jung S. and Li Y. Hyers-Ulam Stability for Second-Order Linear differential Equations with Boundary Conditions, EJDEo 80 (2011) 1-7,

[27] Jung S. and Brzdek J. Hyers-Ulam Stability of the Delay Equation, Abstract and Applied Analysis, Article ID 372176, doi: 10.1155/2010/372176, (2010) 10.

[28] Qusuay H. A, Note on the stability for linear systems of differential equations, International Journal of Applied Mathematical Research 3, 1 (2014) 15-22.

[29] Baleanu D., Guvenc Z., Machado J., New Trends in Nanotechnology and Fractional Calculus Applications, Springer (2010) New York.

[30] Kilbas A., Srivastava M. Trujillo J., Theory and Applications of Fractional Differential Equations, North-Holland Mathematics Studies 204, Elsevier Science B.V. (2006) Amsterdam.

[31] Wang, C. \& Xu, T.Hyers-Ulam stability of fractional linear differential equations involving Caputo fractional derivatives, Appl Math 60 (2015) 383, doi: 10.1007/s10492-0150102-x.

[32] Ibrahim R., Generalized Ulam-Hyers Stability For Fractional Differential Equations, Int. J. Math. 23, 1250056 (2012) 9, doi: 10.1142/S0129167X12500565.

[33] Ibrahim R., Hyers-Ulam stability of fractional linear differential equations in the sense of Caputo Operator, Songklanakarin J. Sci. Technol. 346 (2012) 701-705. 
[34] Barrett J., Differential equations of non-integer order, Canadian J. Math. 6 (1954), 529541.

[35] Plaat O. Ordinary Differential Equations, Holden-Day Series in Mathematics, HoldenDay, (1971) San Francisco. 